\title{
El futuro de la salud pública en el contexto político- social actual
}

\author{
The future of the public health in the political and social \\ setting
}

\section{Camilo González Pérez'; Tatiana Duran Morales"}

\author{
'Máster en Salud Pública. Sede Universitaria Municipal de Playa. La Habana, Cuba. \\ "Máster en Atención Primaria de Salud. Sede Universitaria Municipal de Playa. La \\ Habana, Cuba.
}

\section{RESUMEN}

Organizaciones mundiales de la salud han planteado, desde hace más de 30 años, la necesidad de trabajar para lograr un alto nivel de salud para todos los ciudadanos del planeta. Para alcanzar la meta propuesta de "Salud para todos en el año 2000", se necesita realizar acciones políticas y sociales estrechamente vinculadas, entre ellas pueden citarse: a) el desarrollo social y económico que les permita a los pueblos el derecho al desarrollo sostenible, b) la voluntad política de los gobiernos de priorizar la educación y la salud como uno de los derechos humanos más noble y, c) la transformación revolucionaria de los sistemas de salud, con la intención explícita de colocar a la atención primaria, con los conceptos de promoción y prevención de salud, en la estrategia principal para lograr mejorar los sistemas sanitarios de los pueblos. Sin embargo, a pesar de que el futuro de la salud es una preocupación de prestadores y usuarios de sus servicios, políticos, científicos e intelectuales; puede decirse que en la actualidad tal futuro es incierto, amenazado por las tendencias neoliberales, pero con una realidad alentadora en los países que hoy están optando por el cambio y que se integran al proyecto ALBA. La meta "Salud para todos en el año 2000", está distante aún, todo dependerá de la medida en que el mundo comprenda que la paz, la solidaridad, el respeto a los derechos humanos y la integración de los pueblos, son el único camino para acercarse a esos sueños. 
Palabras clave: Organización Mundial de la Salud, salud pública, atención primaria de salud, promoción y prevención de salud.

\begin{abstract}
Health organizations worldwide have stated for more than 30 years the need of working hard to attain high health levels for all the citizens in this planet. To fulfill the goal "Health for all in the year 2000", closely linked political and social actions are required, among them the following a) the social and economic development that gives the peoples the right to sustainable development; $b$ ) the political willingness of governments to give priority to education and healthcare as two of the noblest human rights and c) the revolutionary transformation of healthcare systems, with the explicit aim of placing the primary healthcare in the forefront of the main strategy for improving the healthcare systems of the countries under the concepts of health promotion and prevention. However, in spite of the fact that the future of healthcare is a permanent concern for health service providers and users, politicians, scientists and intellectuals, it may be said that at present this future is uncertain and threatened by neoliberal tendencies, except for the encouraging realities in those countries opting for change and participating in the ALBA program (Bolivarian Alternative for the Americas). The goal "Health for all in the year 2000" is still far from being accomplished; all this will depend on to what extent the world is able to understand that peace, solidarity, respect to human rights and integration of peoples are the only way to make these dreams come true.
\end{abstract}

Key words: World Health Organization, public health, primary health care, health promotion and prevention.

\title{
I NTRODUCCI ÓN
}

El futuro de la salud es una preocupación de prestadores y usuarios de sus servicios, políticos, científicos e intelectuales. Desde hace más de 30 años en 1977 la Asamblea Mundial de la Salud estableció que la principal meta social de los gobiernos y de la Organización Mundial de la Salud (OMS) debía consistir en alcanzar para todos los ciudadanos del mundo para el año 2000 un grado de salud que les permitiera llevar una vida social y económicamente productiva, es decir, la "Salud para todos en el año 2000."

En 1978, la Conferencia Internacional de Atención Primaria de Salud, celebrada en Alma-Atá, declaró que la atención primaria era la clave para alcanzar esta meta. En 1979, la Asamblea Mundial de la Salud lanzó la estrategia de la salud para todos al hacer suyos el informe y la declaración de Ama-Atá e invitó a todos los estados miembros a que emprendieran individualmente la formulación de estrategias regionales y mundiales. ${ }^{1}$ 


\section{REFLEXIONES}

La meta "Salud para todos en el año 2000", una de las aspiraciones más justas y necesarias que se haya planteado la sociedad, requiere sin dudas acciones estrechamente vinculadas, aunque para su análisis se pueden separar. Estas son:

El desarrollo social y económico que les permita a los pueblos el derecho al desarrollo sostenible. Es por tanto, sin dudas, una necesidad vital que se detenga la carrera armamentista y vale la pena entonces preguntarse una y otra vez ¿Cuántos males y problemas de salud podrían resolverse si se utilizara en acciones de salud las inmensas sumas que los gobiernos gastan en la guerra?

No es un secreto para nadie que el mundo contemporáneo ha seguido la tendencia de los modelos neoliberales que cada vez se alejan más de los sueños de justicia social donde la equidad en la salud o lo que es lo mismo, las aspiraciones de salud para todos cada vez están más lejos de realizarse. Pero es también visible que en Latinoamérica los pueblos cada día luchan por lograr estos sueños y junto al proyecto de la Alternativa Bolivariana para las América (ALBA), se abre una luz a la esperanza de lograrlos.

Ahora bien, el sector de la salud no está ajeno a estos cambios. Las grandes tendencias afectan su organización y la forma de como debe encarar la prestación de sus servicios. En particular, la situación sociopolítica y económica individual de cada país determina no solamente el papel que deberá cumplir este sector, sino que también define sus grandes retos y responsabilidades. ${ }^{2}$

La voluntad política que permita que los gobiernos le den la verdadera prioridad que tiene la educación y la salud como uno de los derechos humanos más noble, es otra de las acciones que se deben realizar.

Como se sustenta claramente al discurrir derechos humanos, todo individuo debe tener derecho a disfrutar del más alto nivel posible de salud física y mental, ya que sin esta posibilidad el individuo no está en capacidad de hacer frente a los avatares de su propia existencia y de luchar en igualdad de condiciones por procurarse una vida digna. Disfrutar de salud de forma tal que permita a los seres humanos vivir dignamente, requiere por parte de los estados, además de suscribir y ratificar oficialmente los tratados internacionales en materia de derechos humanos, desarrollar políticas públicas que materialicen la realización de este derecho. ${ }^{3}$

El Relator Especial del Derecho a la Salud de las Naciones Unidas ha planteado claramente que las normas internacionales de derechos humanos, en particular el derecho a la salud, debe aplicarse de manera coherente y sistemática en todos los procesos de formulación de políticas en los planos nacional e internacional. ${ }^{3}$

En este acápite debe abordarse también el hecho de que según sea el sistema político, es que se definen las políticas sanitarias.

Así el capitalismo y los defensores del modelo privado aseguraban que la atención privada, constituye la respuesta a las dificultades del sector público, incapaz desde el punto de vista financiero para absorber los costos y deficiencias en la gestión y administración de los servicios. No obstante, la liberalización de una economía global de mercado ha llevado a una fuerte privatización y a una concepción cada vez más mercantil de los servicios de salud. El retiro parcial del Estado, la formación de un mercado para el sector privado y el crecimiento de las estructuras para la autonomía y rentabilidad del sector privado en el área de la salud, están 
haciendo de la atención médica un lucrativo negocio, muy lejos de los principios originales y a una distancia sideral de los conceptos elementales del quehacer en salud. $^{4}$

Los defensores del modelo socialista como el de Cuba, defienden la tesis opuesta, como plantea Rojas Ochoa en su artículo publicado en el 2003:

En lo que a salud respecta entonces se concibió y creó el actual sistema de salud, que reconoce esta como un derecho humano y una responsabilidad del estado, que financia el presupuesto de la nación y que alcanza cobertura nacional.

Este sistema se basa en los logros de la ciencia y la técnica, profesa una filosofía humanista, tiene una orientación promotora y preventiva de la salud, y afianza sus raíces en una amplia participación social y en el respaldo de la voluntad política, sostenida a lo largo de más de 40 años.

No obstante la crisis económica y los efectos de la guerra económica, bloqueo incluido, que nos hace el gobierno de los EE.UU., los éxitos de los primeros años se han sostenido y se ha alcanzado mucho más.

Ejemplos concretos: mortalidad infantil muy baja, esperanza de vida al nacer muy alta, analfabetismo erradicado como fenómeno social, liquidación de epidemias en plazos breves, aplicando un modelo que se afirma en la participación popular, conducido por el gobierno a su más alto nivel.

Todo esto expresado en apretada síntesis, encierra una enseñanza: cuando se reúne voluntad política, tecnología adecuada y participación popular, en un escenario libre de corrupción y de presiones externas, se puede alcanzar resultado como lo demostrado por la reforma que hizo la Revolución Cubana. Y no sólo para hacer la reforma, son también las condiciones que conducen a la sostenibilidad de la reforma, que por demás se afianza en sus resultados concretos de mayor equidad y mejoras importantes en el estado de salud de la población, así como en la confianza de la población en su sistema. ${ }^{5}$

La transformación revolucionaria de los sistemas de salud seria otra de las acciones a realizar, que permita colocar a la atención primaria de salud con los conceptos de promoción y prevención de salud, en la estrategia principal para lograr mejorar los sistemas sanitarios de los pueblos, siendo esta sin duda una de las más extraordinarias contribuciones de la OMS en sus 60 años de existencia.

Hoy al conmemorarse 6 años de la declaración de Baleares en defensa de la sanidad pública, sus consideraciones mantienen actual vigencia. Esta declaración considera necesario, en primer lugar, poner fin a las guerras que asolan el planeta, seguir el proceso de debate a escala internacional para desenmascarar las políticas neoliberales y su repercusión sobre la salud y la calidad de vida de las poblaciones, realizar propuestas de reformas de los sistemas sanitarios y sociales públicos que mejoren su nivel de eficacia, eficiencia y calidad en lugar de su destrucción; organizar y coordinar los esfuerzos y la lucha de profesionales y ciudadanos contra las políticas privatizadoras y de destrucción de los sistemas sanitarios públicos.

En la ciudad de San Salvador de Bahía, a los 18 días del mes de julio del año 2007, en ocasión de celebrarse los congresos IV Brasileño de Ciencias Sociales y Humanas en Salud, XIV de la Internacional Association of Health Policy (IAHP) y X de la Asociación Latinoamericana de Medicina Social (ALAMES), esta última se propone desarrollar sus acciones sobre la base de un compromiso social y político dirigido a:

- Fortalecer las estratégias para la defensa de la salud como bien público y derecho ciudadano. 
- Estar conscientes que en el contexto actual el movimiento de la medicina social se caracteriza por el resurgimiento de la lucha antiimperialista en América Latina debido al auge de movimientos sociales y políticos, de gobiernos revolucionarios con un discurso y práctica contrahegemónicos, de un imperialismo norteamericano cuyas acciones se expresan en la agresión militar, terrorista, excluyente, con las consecuencias ineludibles para el deterioro de la salud y de la calidad de vida de las poblaciones.

- Retomar en teoría y práctica las categorías imperialismo, capitalismo, clases sociales y otras como género, etnia y territorio, así como las que se refieren a la defensa del ambiente, la difusión del pensamiento médico social y su utilización como herramienta para una práctica política transformadora.

- Apoyar y acompañar a los países que constituyen la "Punta de lanza" en los avances transformadores de la sociedad, como son Cuba, Venezuela, Ecuador y Bolivia, blancos principales de las agresiones directas del imperialismo, así como Uruguay y Brasil que emergen progresivamente con fuerza en el escenario latinoamericano.

- Apoyar las instancias transformadoras para el desarrollo de Latinoamericana como el ALBA en contra de los acuerdos castrantes para los países del área como el ALCA. ${ }^{6}$

Ahora bien para hablar del futuro de la salud, se debe considerar la conceptualización de desarrollo humano que se presentó en el informe sobre este indicador para el PNUD en 1993, que concuerda más con la justicia social real para todo ser humano:

El desarrollo humano es el desarrollo del pueblo, para el pueblo y por el pueblo. ... del pueblo significa invertir en capacidades humanas, sea en educación o en salud o en aptitudes, con el objetivo de que la gente pueda trabajar de forma productiva y creativa. ...para el pueblo significa asegurar que el crecimiento que genere éste se reparta de modo amplio y justo. ... por el pueblo, es dar a todos una oportunidad de participar. ${ }^{7}$

\section{CONSI DERACI ONES FI NALES}

El futuro de la salud pública es en la actualidad incierto, amenazado por las tendencias neoliberales, pero con una realidad alentadora en los países que hoy están optando por el cambio y que se integran al proyecto ALBA. Las metas de "Salud para todos" están distantes de lograrse, todo dependerá de la medida en que el mundo comprenda que la paz, la solidaridad, el respeto a los derechos humanos, la integración de los pueblos con el concepto de complementariedad; es el único camino para acercarse a estos sueños. Lo anterior debe hacerse consciente más temprano que tarde porque de aquí dependerá el futuro de la salud.

\section{REFERENCI AS BI BLI OGRÁFI CAS}

1. OMS. La 34a Asamblea Mundial de la Salud adopta la estrategia mundial de salud para todos. Crónica de la OMS. Ginebra: OMS; 1981. 
2. Motta PR. Manual sobre Tendencias Contemporáneas en la Gestión de Salud. Vol1. Washington, D.C.: Paltex. OPS/OMS; 1996.[HSP-UNI/Manuales Operativos].

3. Torres Tovar M. El impacto de los acuerdos de libre comercio sobre el derecho a la salud. Rev Cubana Salud Pública [serie en Internet]. 2006 Sep[citado 12 Abr 2008]; 32(3). Disponible en:

http://scielo.sld.cu/scielo.php?script=sci arttext\&pid=S0864 -

34662006000300008\&lng=es\&nrm=iso>

4. Llambias Wolff J. Los desafíos inconclusos de la salud y las reflexiones para el futuro en un mundo globalizado. Rev Cubana Salud Pública. 2003;29(3):236-45.

5. Rojas Ochoa F. El desarrollo de la economía global y su impacto sobre las políticas de salud. Rev Cubana Salud Pública. 2003;29(3):253-9.

6. Declaración de Salvador de Bahía. Rev Cubana Salud Pública [serie en Internet]. 2007[ citado 12 Abril 2008]; 33(4). Disponible en:

http://scielo.sld.cu/scielo.php?script=sci_arttext\&pid=S0864 $\underline{34662007000400021 \& \operatorname{lng}=\mathrm{es} \& n \mathrm{rm}=\mathrm{iso}}$

7. Informe sobre Desarrollo Humano. 1993. PNUD. Madrid: Centro de Comunicación, Investigación, Documentación entre Europa, España y América Latina (CIDEAL); 1993.

Recibido: 15 de mayo de 2008.

Aprobado: 22 de octubre de 2008.

Camilo González Pérez. Sede Universitaria Municipal de Playa. 5ta. Ave. y 14.

Playa. La Habana, Cuba.

E-mail: deenero@infomed.sld.cu 